\title{
Ergodic properties of generalized Lüroth series
}

\author{
by \\ Jose Barrionuevo (Mobile, Ala.), \\ Robert M. Burton (Corvallis, Ore.), \\ Karma Dajani (Utrecht) and Cor KraAikamp (Delft)
}

\section{Introduction}

1.1. Lüroth series. In $1883 \mathrm{~J}$. Lüroth [Lu] introduced and studied the following series expansion, which can be viewed as a generalization of the decimal expansion. Let $x \in(0,1]$. Then

(1) $x=\frac{1}{a_{1}}+\frac{1}{a_{1}\left(a_{1}-1\right) a_{2}}+\ldots+\frac{1}{a_{1}\left(a_{1}-1\right) \ldots a_{n-1}\left(a_{n-1}-1\right) a_{n}}+\ldots$,

where $a_{n} \geq 2, n \geq 1$. Lüroth showed, among other things, that each irrational number has a unique infinite expansion (1), and that each rational number has either a finite or a periodic expansion.

Underlying the series expansion (1) is the operator $T_{L}:[0,1] \rightarrow[0,1]$, defined by

$$
T_{L} x:=\left\lfloor\frac{1}{x}\right\rfloor\left(\left\lfloor\frac{1}{x}\right\rfloor+1\right) x-\left\lfloor\frac{1}{x}\right\rfloor, \quad x \neq 0 ; \quad T 0:=0
$$

(see also Figure 1), where $\lfloor\xi\rfloor$ denotes the greatest integer not exceeding $\xi$. For $x \in[0,1]$ we define $a(x):=\lfloor 1 / x\rfloor+1, x \neq 0 ; a(0):=\infty$ and $a_{n}(x)=$ $a\left(T^{n-1} x\right)$ for $n \geq 1$. From (2) it follows that $T_{L} x=a_{1}\left(a_{1}-1\right) x-\left(a_{1}-1\right)$, and therefore

$$
\begin{aligned}
x & =\frac{1}{a_{1}}+\frac{1}{a_{1}\left(a_{1}-1\right)} T_{L} x \\
& =\frac{1}{a_{1}}+\frac{1}{a_{1}\left(a_{1}-1\right) a_{2}}+\ldots+\frac{T_{L}^{n} x}{a_{1}\left(a_{1}-1\right) \ldots a_{n}\left(a_{n}-1\right)} .
\end{aligned}
$$

Since $a_{n} \geq 2$ and $0 \leq T_{L}^{n} x \leq 1$ one easily sees that the infinite series from (1) converges to $x$.

1991 Mathematics Subject Classification: 28D05, 11K55. 
Recently S. Kalpazidou, A. and J. Knopfmacher introduced and studied in $\left[\mathrm{K}^{3} 1,2\right]$ the so-called alternating Lüroth series. For each $x \in(0,1]$ one has

$$
\begin{aligned}
& x=\frac{1}{a_{1}-1}-\frac{1}{a_{1}\left(a_{1}-1\right)\left(a_{2}-1\right)}+\ldots \\
& \quad+\frac{(-1)^{n+1}}{a_{1}\left(a_{1}-1\right) \ldots a_{n-1}\left(a_{n-1}-1\right)\left(a_{n}-1\right)}+\ldots,
\end{aligned}
$$

where $a_{n} \geq 2, n \geq 1$. Dynamically the alternating series expansion is generated by the operator $S_{A}:[0,1] \rightarrow[0,1]$ defined by

$$
S_{A} x:=1+\left\lfloor\frac{1}{x}\right\rfloor-\left\lfloor\frac{1}{x}\right\rfloor\left(\left\lfloor\frac{1}{x}\right\rfloor+1\right) x, \quad x \neq 0,
$$

and $S_{A} 0:=0$, i.e. $S_{A} x=1-T_{L} x$ (see also Figure 1 ).

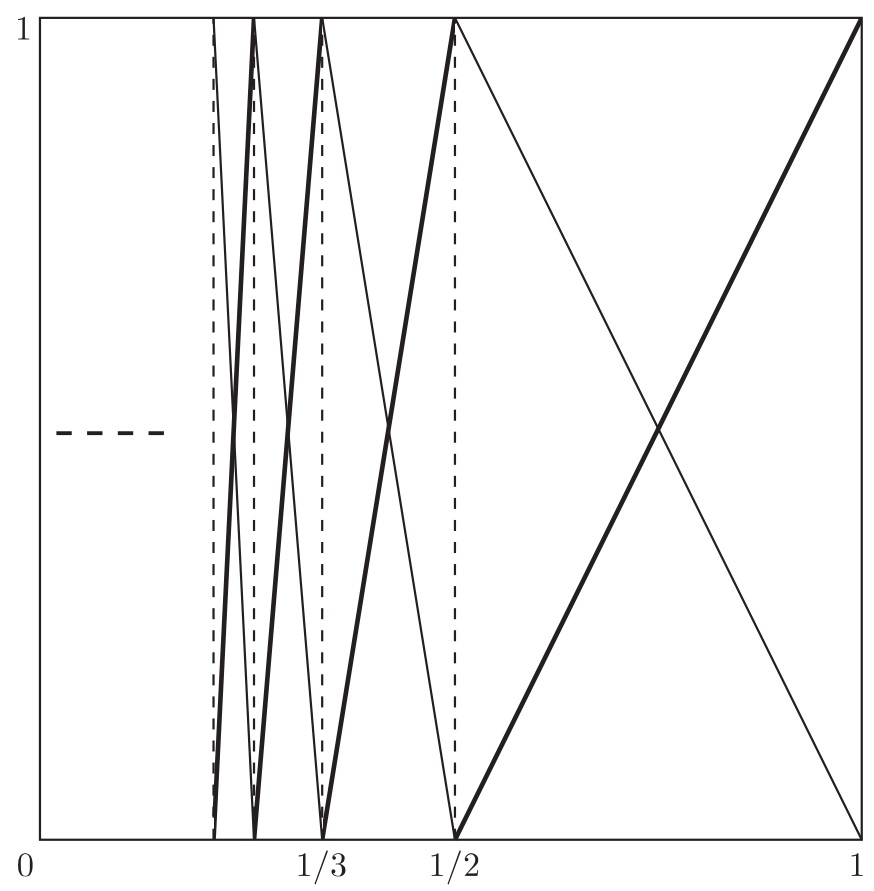

Fig. 1

Lüroth series have been extensively studied; for further reference we mention here papers by H. Jager and C. de Vroedt [JdV], T. Šalát [Sa], and books by J. Galambos [G], O. Perron [Pe] and W. Vervaat [V].

In $[\mathrm{JdV}]$ it was shown that the stochastic variables $a_{1}(x), a_{2}(x), \ldots$ are independent with $\lambda\left(a_{n}=k\right)=1 /(k(k-1))$ for $k \geq 2$, and that $T_{L}$ is 
measure preserving with respect to Lebesgue measure $\left({ }^{1}\right)$ and ergodic $\left({ }^{2}\right)$. Using similar techniques analogous results were obtained in $\left[\mathrm{K}^{3} 1,2\right]$ for the operator $S_{A}$ from (3). In fact, much stronger results can be obtained easily, not only in the case of the (alternating) Lüroth series, but also in a more general setting.

1.2. Generalized Lüroth series. Let $I_{n}=\left(l_{n}, r_{n}\right], n \in \mathcal{D} \subset \mathbb{N}=$ $\{0,1,2, \ldots\}$, be a finite or infinite collection of disjoint intervals of length $L_{n}:=r_{n}-l_{n}$, such that

$$
\sum_{n \in \mathcal{D}} L_{n}=1
$$

and

$$
0<L_{i} \leq L_{j}<1 \quad \text { for all } i, j \in \mathcal{D}, i>j .
$$

The set $\mathcal{D}$ is called the digit set. Usually such a digit set is either a finite or infinite set of consecutive positive integers, see also the examples at the end of this section.

Furthermore, let $I_{\infty}:=[0,1] \backslash \bigcup_{n \in \mathcal{D}} I_{n}, L_{\infty}:=0$ and define the maps $T, S:[0,1] \rightarrow[0,1]$ by

$$
\begin{aligned}
& T x:= \begin{cases}\frac{x-l_{n}}{r_{n}-l_{n}}, & x \in I_{n}, n \in \mathcal{D}, \\
0, & x \in I_{\infty},\end{cases} \\
& S x:= \begin{cases}\frac{r_{n}-x}{r_{n}-l_{n}}, & x \in I_{n}, n \in \mathcal{D}, \\
0, & x \in I_{\infty} .\end{cases}
\end{aligned}
$$

Define for $x \in \Omega:=[0,1] \backslash I_{\infty}=\bigcup_{n \in \mathcal{D}} I_{n}$,

$$
\begin{gathered}
s(x):=\frac{1}{r_{n}-l_{n}} \quad \text { and } \quad h(x):=\frac{l_{n}}{r_{n}-l_{n}}, \quad \text { in case } x \in I_{n}, n \in \mathcal{D}, \\
s_{n}=s_{n}(x):= \begin{cases}s\left(T^{n-1} x\right), & T^{n-1} x \notin I_{\infty}, \\
\infty, & T^{n-1} x \in I_{\infty},\end{cases}
\end{gathered}
$$

and

$$
h_{n}=h_{n}(x):= \begin{cases}h\left(T^{n-1} x\right), & T^{n-1} x \notin I_{\infty} \\ 1, & T^{n-1} x \in I_{\infty}\end{cases}
$$

For $x \in(0,1)$ such that $T^{n-1} x \notin I_{\infty}$, one has

$$
T x=s(x) x-h(x)=s_{1} x-h_{1} .
$$

$\left({ }^{1}\right)$ That is, $\lambda\left(T_{L}^{-1}(A)\right)=\lambda(A)$ for every Lebesgue measurable set $A$.

$\left({ }^{2}\right)$ That is, $\lambda\left(A \triangle T_{L}^{-1} A\right)=0 \Rightarrow \lambda(A) \in\{0,1\}$. 
Inductively we find

(6)

$$
\begin{aligned}
x & =\frac{h_{1}}{s_{1}}+\frac{1}{s_{1}} T x=\frac{h_{1}}{s_{1}}+\frac{1}{s_{1}}\left(\frac{h_{2}}{s_{2}}+\frac{1}{s_{2}} T^{2} x\right)=\ldots \\
& =\frac{h_{1}}{s_{1}}+\frac{h_{2}}{s_{1} s_{2}}+\ldots+\frac{h_{n}}{s_{1} \ldots s_{n}}+\frac{1}{s_{1} \ldots s_{n}} T^{n} x .
\end{aligned}
$$

Since $S x=1-T x=-s_{1} x+1+h_{1}$, for $x \in \Omega$, one finds

$$
x=\frac{h_{1}+1}{s_{1}}-\frac{S x}{s_{1}} .
$$

Continuing in this way we obtain an alternating series expansion (see also $\left.\left[\mathrm{K}^{3} 1,2\right]\right)$. Figure 1 illustrates the case $\mathcal{D}=\mathbb{N} \backslash\{0,1\}, I_{n}:=(1 / n, 1 /(n-1)]$.

Now let $\varepsilon=(\varepsilon(n))_{n \in \mathcal{D}}$ be an arbitrary, fixed sequence of zeroes and ones. We define the map $T_{\varepsilon}:[0,1] \rightarrow[0,1]$ by

$$
T_{\varepsilon} x:=\varepsilon(x) S x+(1-\varepsilon(x)) T x, \quad x \in[0,1],
$$

where

$$
\varepsilon(x):= \begin{cases}\varepsilon(n), & x \in I_{n}, n \in \mathcal{D}, \\ 0, & x \in I_{\infty} .\end{cases}
$$

Let $\varepsilon_{n}:=\varepsilon\left(T_{\varepsilon}^{n-1} x\right)$,

$$
s_{n}=s_{n}(x):= \begin{cases}s\left(T_{\varepsilon}^{n-1} x\right), & T_{\varepsilon}^{n-1} x \notin I_{\infty}, \\ \infty, & T_{\varepsilon}^{n-1} x \in I_{\infty},\end{cases}
$$

and $h_{n}$ defined similarly. By (6) and (7) one finds that

$$
\begin{aligned}
x= & \frac{h_{1}+\varepsilon_{1}}{s_{1}}+\frac{(-1)^{\varepsilon_{1}}}{s_{1}} T_{\varepsilon} x \\
= & \frac{h_{1}+\varepsilon_{1}}{s_{1}}+\frac{(-1)^{\varepsilon_{1}}}{s_{1}}\left(\frac{h_{2}+\varepsilon_{2}}{s_{2}}+\frac{(-1)^{\varepsilon_{2}}}{s_{2}} T_{\varepsilon}^{2} x\right)=\ldots \\
= & \frac{h_{1}+\varepsilon_{1}}{s_{1}}+(-1)^{\varepsilon_{1}} \frac{h_{2}+\varepsilon_{2}}{s_{1} s_{2}}+(-1)^{\varepsilon_{1}+\varepsilon_{2}} \frac{h_{3}+\varepsilon_{3}}{s_{1} s_{2} s_{3}}+\ldots \\
& +(-1)^{\varepsilon_{1}+\ldots+\varepsilon_{n-1}} \frac{h_{n}+\varepsilon_{n}}{s_{1} \ldots s_{n}}+\frac{(-1)^{\varepsilon_{1}+\ldots+\varepsilon_{n}}}{s_{1} \ldots s_{n}} T_{\varepsilon}^{n} x .
\end{aligned}
$$

For each $k \geq 1$ and $1 \leq i \leq k$ one has $s_{i} \geq 1 / L>1$, where $L=\max _{n \in \mathcal{D}} L_{n}$, and $\left|T_{\varepsilon}^{k} x\right| \leq 1$. Thus,

$$
\left|x-\frac{p_{k}}{q_{k}}\right|=\frac{T_{\varepsilon}^{k} x}{s_{1} \ldots s_{k}} \leq L^{k} \rightarrow 0 \quad \text { as } k \rightarrow \infty,
$$

where

$$
\begin{aligned}
\frac{p_{k}}{q_{k}}= & \frac{h_{1}+\varepsilon_{1}}{s_{1}}+(-1)^{\varepsilon_{1}} \frac{h_{2}+\varepsilon_{2}}{s_{1} s_{2}}+(-1)^{\varepsilon_{1}+\varepsilon_{2}} \frac{h_{3}+\varepsilon_{3}}{s_{1} s_{2} s_{3}}+\ldots \\
& +(-1)^{\varepsilon_{1}+\ldots+\varepsilon_{k-1}} \frac{h_{k}+\varepsilon_{k}}{s_{1} \ldots s_{k}}
\end{aligned}
$$


and $q_{k}=s_{1} \ldots s_{k}$. In general $p_{k}$ and $q_{k}$ need not be relatively prime (see also Section 3.1). Let $\varepsilon_{0}:=0$, then for each $x \in[0,1]$ one has

$$
x=\sum_{n=1}^{\infty}(-1)^{\varepsilon_{0}+\ldots+\varepsilon_{n-1}} \frac{h_{n}+\varepsilon_{n}}{s_{1} \ldots s_{n}} .
$$

For each $x \in[0,1]$ we define its sequence of digits $a_{n}=a_{n}(x), n \geq 1$, as follows:

$$
a_{n}=k \Leftrightarrow T_{\varepsilon}^{n-1} x \in I_{k}
$$

for $k \in \mathcal{D} \cup\{\infty\}$. The expansion $(*)$ is called the $(I, \varepsilon)$-generalized Lüroth series $(G L S)$ of $x$. Notice that for each $x \in[0,1] \backslash I_{\infty}$ one finds a unique expansion $(*)$, and therefore a unique sequence of digits $a_{n}, n \geq 1$. Conversely, each sequence of digits $a_{n}, n \geq 1$, with $a_{n} \in \mathcal{D} \cup\{\infty\}$ and $a_{1} \neq \infty$ defines a unique series expansion $(*)$. We denote $(*)$ by

$$
x=\left[\begin{array}{c}
\varepsilon_{1}, \varepsilon_{2}, \varepsilon_{3}, \ldots, \varepsilon_{n}, \ldots \\
a_{1}, a_{2}, a_{3}, \ldots, a_{n}, \ldots
\end{array}\right] .
$$

Since $\varepsilon_{n}=\varepsilon\left(a_{n}\right), n \geq 1$, we might as well replace (11) by

$$
x=\left[a_{1}, a_{2}, a_{3}, \ldots, a_{n}, \ldots\right]
$$

No new information is obtained using (11) instead of (12). However, we will see in Section 3 that it is sometimes adventageous to use (11) instead of (12).

Finite truncations of the series in $(*)$ yield the sequence $p_{n} / q_{n}$ of $(I, \varepsilon)$ GLS convergents of $x$. We denote $p_{n} / q_{n}$ by

$$
\frac{p_{n}}{q_{n}}=\left[\begin{array}{l}
\varepsilon_{1}, \varepsilon_{2}, \varepsilon_{3}, \ldots, \varepsilon_{n} \\
a_{1}, a_{2}, a_{3}, \ldots, a_{n}
\end{array}\right] \text {. }
$$

EXAMPLES. 1. Let $I_{n}:=(1 / n, 1 /(n-1)], n \geq 2$. In case $\varepsilon_{n}=0$ for $n \geq 2$, one gets the classical Lüroth series, while $\varepsilon_{n}=1$ for $n \geq 2$ yields the alternating Lüroth series.

2. For $n \in \mathbb{N}, n \geq 2$, put $I_{i}=(i / n,(i+1) / n], i=0,1, \ldots, n-1$. In case $\varepsilon(i)=0$ for all $i, T_{\varepsilon}$ yields the $n$-adic expansion. In case $n=2$ and $\varepsilon(0)=0$, $\varepsilon(1)=1, T_{\varepsilon}$ is the tent map.

See also Sections 3.2 and 3.3 for more intricate examples.

2. Ergodic properties of generalized Lüroth series. Let $\Omega$ be as in Section $1.2, \mathcal{B}$ be the collection of Borel subsets of $\Omega$, and $\lambda$ be the Lebesgue measure on $(\Omega, \mathcal{B})$. Let $(I, \varepsilon)$ be as in the previous section, viz. $I=\left(I_{n}\right)_{n \in \mathcal{D}}$ satisfies (4) and (5), while $\varepsilon=(\varepsilon(n))_{n \in \mathcal{D}}$ is a sequence of zeroes and ones. We have the following lemma.

Lemma 1. The stochastic variables $a_{1}(x), a_{2}(x), \ldots$, corresponding to the $(I, \varepsilon)-G L S$ operator $T_{\varepsilon}$ from (8) are i.i.d. with respect to the Lebesgue 
measure $\lambda$, and

$$
\lambda\left(a_{n}=k\right)=L_{k} \quad \text { for } k \in \mathcal{D} \cup\{\infty\} .
$$

Furthermore, $\left(I_{n}\right)_{n \in \mathcal{D}}$ is a generating partition.

Proof. Define for $\left(k_{1}, \ldots, k_{n}\right) \in \mathcal{D}^{n}, n \geq 1$, the so-called fundamental intervals of order $n$ by

$$
\Delta_{k_{1} \ldots k_{n}}^{\varepsilon}:=\left\{x \in \Omega: a_{1}(x)=k_{1}, \ldots, a_{n}(x)=k_{n}\right\} .
$$

Let $p_{n} / q_{n} \in \mathbb{Q}$ be defined as in (10) (and recall that the $s_{i}$ 's, $h_{i}$ 's and $\varepsilon_{i}$ 's are uniquely determined by $k_{1}, \ldots, k_{n}$ ), then obviously one has

$$
x \in \Delta_{k_{1} \ldots k_{n}}^{\varepsilon} \Leftrightarrow \exists y \in[0,1] \text { such that } x=\frac{p_{n}}{q_{n}}+\frac{(-1)^{\varepsilon_{1}+\ldots+\varepsilon_{n}}}{s_{1} \ldots s_{n}} y .
$$

Thus $\Delta_{k_{1} \ldots k_{n}}^{\varepsilon}$ is an interval with $p_{n} / q_{n}$ as one endpoint, and having length $1 /\left(s_{1} \ldots s_{n}\right)$. Therefore,

$$
\lambda\left(\Delta_{k_{1} \ldots k_{n}}^{\varepsilon}\right)=\lambda\left(a_{1}=k_{1}, \ldots, a_{n}=k_{n}\right)=\frac{1}{s_{1} \ldots s_{n}} .
$$

Since

one finds

$$
s_{i}=\frac{1}{r_{k_{i}}-l_{k_{i}}}=\frac{1}{L_{k_{i}}}, \quad i=1, \ldots, n
$$

$$
\lambda\left(\Delta_{k_{1} \ldots k_{n}}^{\varepsilon}\right)=\lambda\left(a_{1}=k_{1}, \ldots, a_{n}=k_{n}\right)=\prod_{i=1}^{n} L_{k_{i}} .
$$

The independence of the $a_{n}(x)$ 's and the equality $\lambda\left(a_{n}=k\right)=L_{k}$ for $k \in \mathcal{D} \cup\{\infty\}$ now easily follow from

$$
\sum_{k_{i} \in \mathcal{D}} L_{k_{i}}=1 \quad \text { for all } n \geq 1 \text { and all } 1 \leq i \leq n .
$$

That $\left(I_{n}\right)_{n \in \mathcal{D}}$ is a generating partition is immediate from (9).

TheOREM 1. The $(I, \varepsilon)$-GLS operator $T_{\varepsilon}$ from (8) is measure preserving with respect to Lebesgue measure and Bernoulli.

Proof. For any $k_{1}, \ldots, k_{n} \in \mathcal{D}, n \geq 1$,

$$
T_{\varepsilon}^{-1} \Delta_{k_{1} \ldots k_{n}}^{\varepsilon}=\bigcup_{k \in \mathcal{D}} \Delta_{k k_{1} \ldots k_{n}}^{\varepsilon}
$$

is a disjoint union of fundamental intervals of order $n+1$, so that

$$
\begin{aligned}
\lambda\left(T_{\varepsilon}^{-1} \Delta_{k_{1} \ldots k_{n}}^{\varepsilon}\right) & =\sum_{k \in \mathcal{D}} \lambda\left(\Delta_{k k_{1} \ldots k_{n}}^{\varepsilon}\right)=L_{k_{1}} \ldots L_{k_{n}} \sum_{k \in \mathcal{D}} L_{k} \\
& =L_{k_{1}} \ldots L_{k_{n}}=\lambda\left(\Delta_{k_{1} \ldots k_{n}}^{\varepsilon}\right) .
\end{aligned}
$$

Since the collection $\left\{\Delta_{k_{1} \ldots k_{n}}^{\varepsilon}: n \geq 1, k_{i} \in \mathcal{D}\right\}$ generates $\mathcal{B}$, it follows from $\left[\mathrm{W}\right.$, Theorem 1.1, p. 20] that $\lambda$ is $T_{\varepsilon}$-invariant. From Lemma 1, viz. 


$$
\lambda\left(\Delta_{k_{1} \ldots k_{n}}^{\varepsilon}\right)=\prod_{i=1}^{n} \lambda\left(\Delta_{k_{i}}^{\varepsilon}\right),
$$

we conclude that $\left([0,1], \mathcal{B}, \lambda, T_{\varepsilon}\right)$ is a Bernoulli system.

Remarks. 1. The Bernoullicity of the Lüroth operator $T_{L}$ was already noticed by $\mathrm{P}$. Liardet in $[\mathrm{Li}]$.

2. From the fact that $T_{\varepsilon}$ is Bernoulli, and therefore ergodic, one can draw a great number of easy consequences, using Birkhoff's Ergodic Theorem. See also $[\mathrm{JdV}]$ and $\left[\mathrm{K}^{3} 2\right]$. We mention here some results:

For almost every $x$ the sequence $\left(T_{\varepsilon}^{n} x\right)_{n=0}^{\infty}$ is uniformly distributed over $[0,1]$. Furthermore,

$$
\lim _{n \rightarrow \infty} \frac{1}{n} \sum_{k=0}^{n-1} T_{\varepsilon}^{k} x=\frac{1}{2}, \quad \lim _{n \rightarrow \infty}\left(\prod_{k=0}^{n-1} T_{\varepsilon}^{k} x\right)^{1 / n}=\frac{1}{e} \text { a.e. }
$$

and

$$
\lim _{n \rightarrow \infty}\left(a_{1} \ldots a_{n}\right)^{1 / n}=e^{c} \quad \text { a.e. },
$$

where $c=\sum_{k \in \mathcal{D}} L_{k} \log k\left({ }^{3}\right)$.

Define the map $\mathcal{T}_{\varepsilon}:[0,1] \times[0,1] \rightarrow[0,1] \times[0,1]$ by

$$
\mathcal{T}_{\varepsilon}(x, y):=\left(T_{\varepsilon}(x), \frac{h(x)+\varepsilon(x)}{s(x)}+\frac{(-1)^{\varepsilon(x)}}{s(x)} y\right) .
$$

Notice that for

one has

$$
x=\left[\begin{array}{l}
\varepsilon_{1}, \varepsilon_{2}, \varepsilon_{3}, \ldots, \varepsilon_{n}, \ldots \\
a_{1}, a_{2}, a_{3}, \ldots, a_{n}, \ldots
\end{array}\right]
$$

where

$$
\mathcal{T}_{\varepsilon}(x, 0)=\left(\left[\begin{array}{c}
\varepsilon_{2}, \varepsilon_{3}, \ldots, \varepsilon_{n}, \ldots \\
a_{2}, a_{3}, \ldots, a_{n}, \ldots
\end{array}\right],\left[\begin{array}{c}
\varepsilon_{1} \\
a_{1}
\end{array}\right]\right)
$$

$$
\left[\begin{array}{l}
\varepsilon_{1} \\
a_{1}
\end{array}\right]=(-1)^{\varepsilon_{0}} \frac{h_{1}+s_{1}}{s_{1}}=\frac{h_{1}+s_{1}}{s_{1}} .
$$

Now

where

$$
\mathcal{T}_{\varepsilon}^{2}(x, 0)=\left(\left[\begin{array}{c}
\varepsilon_{3}, \varepsilon_{4}, \ldots, \varepsilon_{n}, \ldots \\
a_{3}, a_{4}, \ldots, a_{n}, \ldots
\end{array}\right],\left[\begin{array}{c}
\varepsilon_{2}, \varepsilon_{1} \\
a_{2}, a_{1}
\end{array}\right]\right),
$$

$\left[\begin{array}{ll}\varepsilon_{2}, & \varepsilon_{1} \\ a_{2}, & a_{1}\end{array}\right]=(-1)^{\varepsilon_{0}} \frac{h_{2}+\varepsilon_{2}}{s_{2}}+(-1)^{\varepsilon_{0}+\varepsilon_{2}} \frac{h_{1}+\varepsilon_{1}}{s_{1} s_{2}}=\frac{h_{2}+\varepsilon_{2}}{s_{2}}+(-1)^{\varepsilon_{2}} \frac{h_{1}+\varepsilon_{1}}{s_{1} s_{2}}$.

$\left({ }^{3}\right)$ In case $0 \in \mathcal{D}$ we put $e^{c}:=0$. 
Putting $\mathcal{T}_{\varepsilon}^{n}(x, 0)=:\left(T_{n}, V_{n}\right), n \geq 0$, where

$$
T_{n}=T_{\varepsilon}^{n} x=\left[\begin{array}{l}
\varepsilon_{n+1}, \varepsilon_{n+2}, \ldots \\
a_{n+1}, a_{n+2}, \ldots
\end{array}\right], \quad n \geq 0,
$$

and

$$
V_{n}=\left[\begin{array}{l}
\varepsilon_{n}, \varepsilon_{n-1}, \ldots, \varepsilon_{1} \\
a_{n}, a_{n-1}, \ldots, a_{1}
\end{array}\right], \quad n \geq 1, \quad V_{0}:=0,
$$

we see inductively that

$$
V_{n+1}=\frac{h_{n+1}+\varepsilon_{n+1}}{s_{n+1}}+\frac{(-1)^{\varepsilon_{n+1}}}{s_{n+1}} V_{n} .
$$

As in the case of the continued fraction we will call $T_{n}=T_{\varepsilon}^{n} x$ the future of $x$ at time $n$, while $V_{n}=V_{n}(x)$ is the past of $x$ at time $n$ (see also $[\mathrm{K}]$ ). We have the following theorem.

Theorem 2. The system $\left([0,1] \times[0,1], \mathcal{B} \times \mathcal{B}, \lambda \times \lambda, \mathcal{T}_{\varepsilon}\right)$ is the natural extension of $\left([0,1], \mathcal{B}, \lambda, T_{\varepsilon}\right)$. Furthermore, $\left([0,1] \times[0,1], \mathcal{B} \times \mathcal{B}, \lambda \times \lambda, \mathcal{T}_{\varepsilon}\right)$ is Bernoulli.

Proof. For any two vectors $\left(k_{1}, \ldots, k_{n}\right) \in \mathcal{D}^{n},\left(l_{1}, \ldots, l_{m}\right) \in \mathcal{D}^{m}$ one has

$$
\Delta_{k_{1} \ldots k_{n}}^{\varepsilon} \times \Delta_{l_{1} \ldots l_{m}}^{\varepsilon}=\mathcal{T}_{\varepsilon}^{m}\left(\Delta_{l_{m} \ldots l_{1} k_{1} \ldots k_{n}}^{\varepsilon} \times[0,1]\right) .
$$

Since $\left\{\Delta_{k_{1} \ldots k_{n}}^{\varepsilon} \times \Delta_{l_{1} \ldots l_{m}}^{\varepsilon}: k_{i}, l_{j} \in \mathcal{D}, 1 \leq i \leq n, 1 \leq j \leq m, n, m \geq 1\right\}$ generates $\mathcal{B} \times \mathcal{B}$, it follows that

$$
\bigvee_{m \geq 0} \mathcal{T}_{\varepsilon}^{m}(\mathcal{B} \times[0,1])=\mathcal{B} \times \mathcal{B}
$$

Now, for any $\Delta_{k_{1} \ldots k_{n}}^{\varepsilon} \times \Delta_{l_{1} \ldots l_{m}}^{\varepsilon}$ one has

$$
\mathcal{T}_{\varepsilon}^{-1}\left(\Delta_{k_{1} \ldots k_{n}}^{\varepsilon} \times \Delta_{l_{1} \ldots l_{m}}^{\varepsilon}\right)=\Delta_{l_{1} k_{1} \ldots k_{n}}^{\varepsilon} \times \Delta_{l_{2} \ldots l_{m}}^{\varepsilon} .
$$

Thus,

$$
\begin{aligned}
\lambda \times \lambda\left(\mathcal{T}_{\varepsilon}^{-1}\left(\Delta_{k_{1} \ldots k_{n}}^{\varepsilon} \times \Delta_{l_{1} \ldots l_{m}}^{\varepsilon}\right)\right) & =\lambda\left(\Delta_{l_{1} k_{1} \ldots k_{n}}^{\varepsilon}\right) \lambda\left(\Delta_{l_{2} \ldots l_{m}}^{\varepsilon}\right) \\
& =\lambda\left(\Delta_{k_{1} \ldots k_{n}}^{\varepsilon}\right) \lambda\left(\Delta_{l_{1} \ldots l_{m}}^{\varepsilon}\right) \\
& =\lambda \times \lambda\left(\Delta_{k_{1} \ldots k_{n}}^{\varepsilon} \times \Delta_{l_{1} \ldots l_{m}}^{\varepsilon}\right) .
\end{aligned}
$$

Since cylinders of the form $\Delta_{k_{1} \ldots k_{n}}^{\varepsilon} \times \Delta_{l_{1} \ldots l_{m}}^{\varepsilon}$ generate the $\sigma$-algebra $\mathcal{B} \times \mathcal{B}$, it follows that $\mathcal{T}_{\varepsilon}$ is measure preserving with respect to Lebesgue measure. Thus, $\mathcal{T}_{\varepsilon}$ is the natural extension of $T_{\varepsilon}$ (see $[R]$ for details). Since $T_{\varepsilon}$ is Bernoulli it is an exercise to show that $\mathcal{T}_{\varepsilon}$ is Bernoulli (see also [B]).

Corollary 1. For almost all $x$ the two-dimensional sequence

$$
\mathcal{T}_{\varepsilon}^{n}(x, 0)=\left(T_{n}, V_{n}\right), \quad n \geq 0,
$$

is uniformly distributed over $[0,1] \times[0,1]$. 
Proof. Denote by A that subset of $[0,1]$ for which the sequence $\left(T_{n}, V_{n}\right)_{n=0}^{\infty}$ is not uniformly distributed over $[0,1] \times[0,1]$. It follows from Lemma 1 and the definition of $\mathcal{T}_{\varepsilon}$ that for all $x, y, y^{*} \in[0,1]$ one has

$$
\left|\mathcal{T}_{\varepsilon}^{n}(x, y)-\mathcal{T}_{\varepsilon}^{n}\left(x, y^{*}\right)\right|<L^{n}, \quad n \geq 0,
$$

and we see that $\left(\mathcal{T}_{\varepsilon}^{n}(x, y)-\mathcal{T}_{\varepsilon}^{n}\left(x, y^{*}\right)\right)_{n=0}^{\infty}$ is a null-sequence. Hence, if $\mathcal{A}:=$ $\mathrm{A} \times[0,1]$, then for every pair $(x, y) \in \mathcal{A}$ the sequence $\mathcal{T}_{\varepsilon}^{n}(x, y), n \geq 0$, is not uniformly distributed over $[0,1] \times[0,1]$. Now, if $\mathrm{A}$ had, as a subset of $[0,1]$, positive Lebesgue measure, so would $\mathcal{A}$ as a subset of $[0,1] \times[0,1]$. However, this is impossible in view of Theorem 2 .

The partition $\xi=\left\{I_{k} \times[0,1]\right\}_{k \in \mathcal{D}}$ is a generator for $\mathcal{T}_{\varepsilon}$, which implies that the entropy $h\left(\mathcal{T}_{\varepsilon}\right)$ of $\mathcal{T}_{\varepsilon}$ equals $h\left(\mathcal{T}_{\varepsilon}, \xi\right)$ (see also [W], p. 96). Therefore,

$$
h\left(\mathcal{T}_{\varepsilon}\right)=-\sum_{k \in \mathcal{D}} L_{k} \log L_{k} .
$$

Now let $\left(I_{k}\right)_{k \in \mathcal{D}}$ and $\left(I_{k}^{*}\right)_{k \in \mathcal{D}}$ be two partitions of $[0,1]$ satisfying (4) and (5), and suppose that $L_{k}=L_{k}^{*}$ for $k \in \mathcal{D}$. Furthermore, let $\varepsilon=\left(\varepsilon_{k}\right)_{k \in \mathcal{D}}$ and $\varepsilon^{*}=\left(\varepsilon_{k}^{*}\right)_{k \in \mathcal{D}}$ be two arbitrary sequences of zeroes and ones. It follows at once from Ornstein's Isomorphism Theorem (see [W], p. 105) and Theorem 2 that $\mathcal{T}_{\varepsilon}$ and $\mathcal{T}_{\varepsilon^{*}}$ are metrically isomorphic. We conclude this section with the following theorem, which gives a concrete isomorphism.

Theorem 3. Let $\left(I_{k}\right)_{k \in \mathcal{D}}$ and $\left(I_{k}^{*}\right)_{k \in \mathcal{D}}$ be two partitions of $[0,1]$, satisfying (4) and (5). Suppose that $L_{k}=L_{k}^{*}$ for $k \in \mathcal{D}$. Furthermore, let $\varepsilon=\left(\varepsilon_{k}\right)_{k \in \mathcal{D}}$ and $\varepsilon^{*}=\left(\varepsilon_{k}^{*}\right)_{k \in \mathcal{D}}$ be two sequences of zeroes and ones. Let $\mathcal{T}_{\varepsilon}$ and $\mathcal{T}_{\varepsilon^{*}}$ be defined as in (8). Finally, define $\Psi:[0,1] \times[0,1] \rightarrow[0,1] \times[0,1]$ by

$$
\begin{aligned}
& \Psi\left(\left[\begin{array}{l}
\varepsilon_{1}, \varepsilon_{2}, \ldots \\
a_{1}, a_{2}, \ldots
\end{array}\right],\left[\begin{array}{l}
\varepsilon_{0}, \varepsilon_{-1}, \ldots \\
a_{0}, a_{-1}, \ldots
\end{array}\right]\right)
\end{aligned}
$$

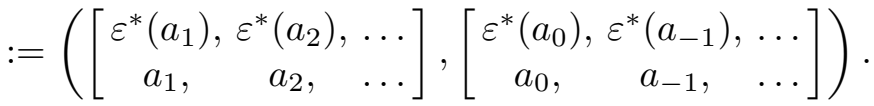

Then $\Psi$ is a measure preserving isomorphism.

Proof. Since almost every $x \in[0,1]$ has unique $(I, \varepsilon)-,\left(I^{*}, \varepsilon^{*}\right)$-GLS expansions, it follows that $\Psi$ is injective. Now, for any cylinder $\Delta_{k_{1} \ldots k_{n}}^{\varepsilon^{*}} \times$ $\Delta_{l_{1} \ldots l_{m}}^{\varepsilon^{*}}$,

$$
\Delta_{k_{1} \ldots k_{n}}^{\varepsilon} \times \Delta_{l_{1} \ldots l_{m}}^{\varepsilon}=\Psi^{-1}\left(\Delta_{k_{1} \ldots k_{n}}^{\varepsilon^{*}} \times \Delta_{l_{1} \ldots l_{m}}^{\varepsilon^{*}}\right)
$$

and 


$$
\begin{aligned}
(\lambda \times \lambda)\left(\Delta_{k_{1} \ldots k_{n}}^{\varepsilon^{*}} \times \Delta_{l_{1} \ldots l_{m}}^{\varepsilon^{*}}\right) & =L_{k_{1}}^{*} \ldots L_{k_{n}}^{*} L_{l_{1}}^{*} \ldots L_{l_{m}}^{*} \\
& =L_{k_{1}} \ldots L_{k_{n}} L_{l_{1}} \ldots L_{l_{m}} \\
& =(\lambda \times \lambda)\left(\Delta_{k_{1} \ldots k_{n}}^{\varepsilon} \times \Delta_{l_{1} \ldots l_{m}}^{\varepsilon}\right) \\
& =(\lambda \times \lambda) \Psi^{-1}\left(\Delta_{k_{1} \ldots k_{n}}^{\varepsilon^{*}} \times \Delta_{l_{1} \ldots l_{m}}^{\varepsilon^{*}}\right) .
\end{aligned}
$$

This shows that $\Psi$ is measurable and measure preserving.

Finally, let $(x, y) \in[0,1] \times[0,1]$ with

$$
x=\left[\begin{array}{c}
\varepsilon_{1}, \varepsilon_{2}, \ldots \\
a_{1}, a_{2}, \ldots
\end{array}\right], \quad y=\left[\begin{array}{c}
\varepsilon_{0}, \varepsilon_{-1}, \ldots \\
a_{0}, a_{-1}, \ldots
\end{array}\right] .
$$

Then

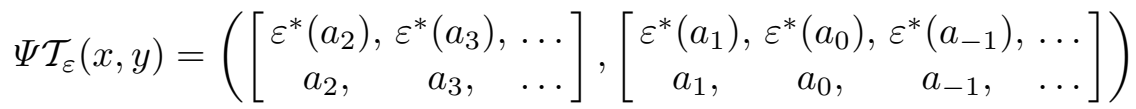

$$
\begin{aligned}
& =\mathcal{T}_{\varepsilon^{*}} \Psi(x, y),
\end{aligned}
$$

therefore $\Psi$ is a measure preserving isomorphism.

\section{Applications and examples}

3.1. Approximation coefficients and their distribution. As before let $I=$ $\left(I_{n}\right)_{n \in \mathcal{D}}$ be a partition of $[0,1]$ which satisfies (4) and (5), and let $\varepsilon=$ $(\varepsilon(n))_{n \in \mathcal{D}}$ be a sequence of zeroes and ones. Putting $q_{k}=s_{1} \ldots s_{k}$, it follows from (9) and Corollary 1 that for a.e. $x$ the approximation coefficients $\theta_{n}$, $n \geq 0$, defined by

$$
\theta_{\varepsilon, n}=\theta_{\varepsilon, n}(x):=q_{n}\left|x-\frac{p_{n}}{q_{n}}\right|, \quad n \geq 0,
$$

have the same distribution as $T_{\varepsilon}^{n} x, n \geq 0$. Viz., for a.e. $x$ the sequence $\left(\theta_{\varepsilon, n}\right)_{n}$ is uniformly distributed on $[0,1]$.

In fact, for many partitions $\left(I_{n}\right)_{n \in \mathcal{D}}$ more information on the distribution of the $\theta_{n}$ 's can be obtained by a more careful definition of $q_{n}$. As an example we will treat here the case of the classical Lüroth series, and all other GLS expansions with the same partition $\left(I_{n}\right)_{n \in \mathcal{D}}$ (see also the examples at the end of Section 1.2).

In this case

$$
s_{n}=s\left(T_{\varepsilon}^{n-1} x\right)=\frac{1}{\frac{1}{a_{n}-1}-\frac{1}{a_{n}}}=a_{n}\left(a_{n}-1\right), \quad h_{n}=a_{n}-1,
$$

and

$$
\frac{h_{n}+\varepsilon_{n}}{s_{1} \ldots s_{n}}=\frac{a_{n}-1+\varepsilon_{n}}{a_{1}\left(a_{1}-1\right) a_{2}\left(a_{2}-1\right) \ldots a_{n}\left(a_{n}-1\right)}=\frac{1}{a_{1}\left(a_{1}-1\right) \ldots\left(a_{n}-\varepsilon_{n}\right)} .
$$

Therefore it is more appropriate to put

$q_{1}=a_{1}-\varepsilon_{1}, \quad q_{n}=a_{1}\left(a_{1}-1\right) a_{2}\left(a_{2}-1\right) \ldots a_{n-1}\left(a_{n-1}-1\right)\left(a_{n}-\varepsilon_{n}\right), \quad n \geq 2$, 
and we see

$$
\theta_{n}(x)=\frac{a_{n}-\varepsilon_{n}}{a_{n}\left(a_{n}-1\right)} T_{\varepsilon}^{n} x, \quad n \geq 1 .
$$

We have the following theorem.

THEOREM 4. Let $\left(I_{n}\right)_{n \in \mathcal{D}}$ be the Lüroth partition, that is, $I_{n}:=(1 / n$, $1 /(n-1)]$ for $n \geq 2$, and let $\varepsilon(n) \in\{0,1\}$ for $n \geq 2$. Then for a.e. $x$ and for every $z \in(0,1]$ the limit

$$
\lim _{N \rightarrow \infty} \frac{1}{N} \#\left\{1 \leq j \leq N: \theta_{j}(x)<z\right\}
$$

exists and equals $F_{\varepsilon}(z)$, where

$$
\begin{aligned}
& F_{\varepsilon}(z):=\sum_{k=2}^{\lfloor 1 / z\rfloor+1-\varepsilon(\lfloor 1 / z\rfloor+1)} \frac{z}{k-\varepsilon(k)}+\frac{1}{\lfloor 1 / z\rfloor+1-\varepsilon(\lfloor 1 / z\rfloor+1)}, \\
& 0<z \leq 1 \text {. }
\end{aligned}
$$

Proof. Let $z \in(0,1]$. From (15) and (16) it follows that

$$
\theta_{n}<z \Leftrightarrow\left(T_{n}, V_{n}\right) \in \Xi(z)=\bigcup_{k=2}^{\infty} \Xi_{k}(z),
$$

where

$$
\Xi_{k}(z):=\left(\left[0, \frac{k(k-1)}{k-\varepsilon(k)} z\right] \cap[0,1]\right) \times \Delta_{k}, \quad k \geq 2 .
$$

For $k \geq 2$ we have the following two cases (of which the first one might be void).

(A) $2 \leq k \leq\lfloor 1 / z\rfloor+1-\varepsilon(\lfloor 1 / z\rfloor+1)$. In this case

$$
\Xi_{k}(z)=\left[0, \frac{k(k-1)}{k-\varepsilon(k)} z\right] \times \Delta_{k} .
$$

(B) $k>\lfloor 1 / z\rfloor+1-\varepsilon(\lfloor 1 / z\rfloor+1)$. In this case $\Xi_{k}(z)=[0,1] \times \Delta_{k}$.

From (A) and (B) one finds, that

$$
\begin{aligned}
(\lambda \times \lambda)(\Xi(z)) & \sum_{k=2}^{\lfloor 1 / z\rfloor+1-\varepsilon(\lfloor 1 / z\rfloor+1)}(\lambda \times \lambda)\left(\left[0, \frac{k(k-1)}{k-\varepsilon(k)} z\right] \times \Delta_{k}\right) \\
& +\frac{1}{\lfloor 1 / z\rfloor+1-\varepsilon(\lfloor 1 / z\rfloor+1)} \\
= & \sum_{k=2}^{\lfloor 1 / z\rfloor+1-\varepsilon(\lfloor 1 / z\rfloor+1)} \frac{z}{k-\varepsilon(k)}+\frac{1}{\lfloor 1 / z\rfloor+1-\varepsilon(\lfloor 1 / z\rfloor+1)} .
\end{aligned}
$$

The theorem at once follows from Corollary 1. 
Remarks. 1. Although the map $x \rightarrow(1 / x)$ mod 1 generating the continued fraction is not piecewise linear, which leads to complications in estimations, a similar result as in Theorem 4 was obtained for continued fractions (see also [BJW]).

2. If $\varepsilon(n)=0, n \geq 2$ (the classical Lüroth case) $\left(^{4}\right)$, the distribution function $F_{\varepsilon}$ reduces to

$$
F_{\mathrm{L}}(z)=\sum_{k=2}^{\lfloor 1 / z\rfloor+1} \frac{z}{k}+\frac{1}{\lfloor 1 / z\rfloor+1}, \quad 0<z \leq 1 .
$$

Furthermore

$$
F_{\mathrm{A}}(z)=\sum_{k=2}^{\lfloor 1 / z\rfloor} \frac{z}{k-1}+\frac{1}{\lfloor 1 / z\rfloor}, \quad 0<z \leq 1 ;
$$

see also Figure 2. Notice that $F_{\mathrm{A}} \leq F_{\varepsilon} \leq F_{\mathrm{L}}$.

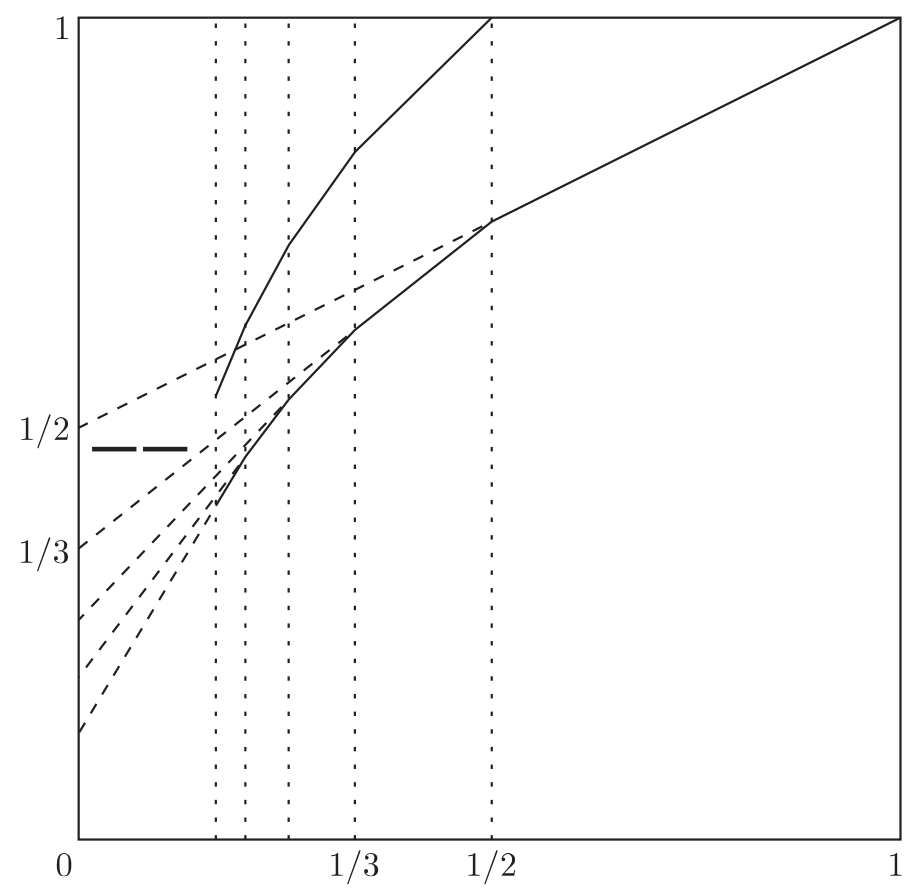

Fig. 2

We have the following corollary.

$\left({ }^{4}\right)$ From now on the classical (resp. the alternating) Lüroth case will be indicated by a subscript L (resp. A). 
Corollary 2. Let $\left(I_{n}\right)_{n \in \mathcal{D}}$ be the Lüroth partition and let $\varepsilon(n) \in\{0,1\}$ for $n \geq 2$. Then there exists a constant $M_{\varepsilon}$ such that for a.e. $x$,

$$
\lim _{N \rightarrow \infty} \frac{1}{N} \sum_{i=1}^{N} \theta_{\varepsilon, i}=M_{\varepsilon} .
$$

Moreover, $M_{\varepsilon}$ can be calculated explicitly, and $M_{\mathrm{A}} \leq M_{\varepsilon} \leq M_{\mathrm{L}}$, where $M_{\mathrm{A}}=1-\frac{1}{2} \zeta(2)=0.177533 \ldots$ and $M_{\mathrm{L}}=\frac{1}{2}(\zeta(2)-1)=0.322467 \ldots$

Proof. By definition $M_{\varepsilon}$ is the first moment of $F_{\varepsilon}$ and thus $M_{\varepsilon}=$ $\int_{0}^{1}\left(1-F_{\varepsilon}(x)\right) d x$.

Remarks. 1. From Corollary 2 it follows that among all $(I, \varepsilon)$-GLS expansions with $I$ the Lüroth partition the alternating Lüroth series has the best approximation properties.

2. The presentation of Corollary 2 suggests that by choosing $\varepsilon=$ $(\varepsilon(n))_{n \geq 2}$ appropriately, each value in the interval $\left[M_{\mathrm{A}}, M_{\mathrm{L}}\right]=[0.177533 \ldots$, $0.322467 \ldots$... might be attained. This is certainly incorrect, as the following example shows. Let $\varepsilon=(\varepsilon(n))_{n \geq 2}$ be given by $\varepsilon(2)=1$ and $\varepsilon(n)=0$ for $n \geq 3$, and let $\varepsilon^{*}=\left(\varepsilon^{*}(n)\right)_{n \geq 2}$ be given by $\varepsilon^{*}(2)=0$ and $\varepsilon^{*}(n)=1$ for $n \geq 3$. A simple calculation yields that $M_{\varepsilon}=M_{\mathrm{L}}-1 / 8=0.197467 \ldots$ and $M_{\varepsilon^{*}}=M_{\mathrm{A}}+1 / 8=0.302533 \ldots$; we thus see that $M_{\varepsilon}<M_{\varepsilon^{*}}$ and from this one can easily deduce that there does not exist a sequence $\varepsilon^{b}=\left(\varepsilon^{b}(n)\right)_{n \geq 2}$ of zeroes and ones for which $M_{\varepsilon^{b}} \in\left[M_{\varepsilon}, M_{\varepsilon^{*}}\right]$. Some further investigations even suggest that the set

$$
\Upsilon:=\left\{M_{\varepsilon}: \varepsilon(n) \in\{0,1\} \text { for } n \geq 2\right\}
$$

is a Cantor set.

3.2. Jump transformations. Let $T_{\varepsilon}$ be a $(I, \varepsilon)$-GLS operator with digit set $\mathcal{D}$, and let $a \in \mathcal{D}$. For $x \in \Omega$, put

$$
n_{a}=n_{a}(x):=\min _{n \geq 1}\left\{a_{n}(x): a_{n}(x)=a\right\}
$$

(and $n_{a}=\infty$ in case $a_{n}(x) \neq a$ for all $n \geq 1$ ). Define the jump transformation $J_{a}: \Omega \rightarrow \Omega$ by

$$
J_{a} x:= \begin{cases}T_{\varepsilon}^{n_{a}} x, & n_{a} \in \mathbb{N} \\ 0, & n_{a}=\infty\end{cases}
$$

Jump transformations were first studied by H. Jager $[\mathrm{J}]$ for the particular case that $T_{\varepsilon} x=10 x(\bmod 1)$. Jager showed that such jump transformations are stronly mixing. Here, in the more general setting, we have a stronger result.

TheOREM 5. Let $T_{\varepsilon}$ be an $(I, \varepsilon)-G L S$ operator with digit set $\mathcal{D}$. For each $a \in \mathcal{D}$ the corresponding jump transformation $J_{a}$, as defined in (18), is an 
$\left(I^{*}, \varepsilon^{*}\right)-G L S$ operator, with

$$
I^{*}=\left\{\Delta_{a_{1} \ldots a_{n}}: n \geq 1, a_{n}=a \text { and } a_{i} \neq a \text { for } 1 \leq i \leq n-1\right\}
$$

and for each $\Delta_{a_{1} \ldots a_{n}} \in I^{*}$ the corresponding value of $\varepsilon$ is given by

$$
\varepsilon^{*}\left(\Delta_{a_{1} \ldots a_{n}}\right)=\varepsilon\left(a_{1}\right)+\ldots+\varepsilon\left(a_{n}\right)(\bmod 2) .
$$

3.3. $\beta$-expansions and pseudo golden mean numbers. For an irrational number $\beta>1$ the $\beta$-transformation $T_{\beta}:[0,1] \rightarrow[0,1]$ is defined by

$$
T_{\beta} x=\beta x(\bmod 1)
$$

(see also [FS] for further references). Clearly, $\beta$-transformations do not belong to the class of GLS-transformations. However, in some cases there exists an intimate relation between both types of transformations, as the following example shows.

Let $\beta>1$ be the positive root of the polynomial $X^{m}-X^{m-1}-\ldots-X-1$, where $\left({ }^{5}\right) m \geq 2$. Due to C. Frougny and B. Solomyak [FS] we know that such $\beta$ 's are Pisot numbers and that the $\beta$-expansion $d(1, \beta)$ is finite, and equals

$$
1=\frac{1}{\beta}+\frac{1}{\beta^{2}}+\ldots+\frac{1}{\beta^{m}} .
$$

Notice that $T_{\beta}^{i} 1=\beta^{-1}+\ldots+\beta^{-(m-i)}, 0 \leq i \leq m-1$, and $T_{\beta}^{i} 1=0$ for $i \geq m$. Furthermore, let

$$
X:=\bigcup_{k=0}^{m-1}\left(T_{\beta}^{m-k} 1, T_{\beta}^{m-k-1} 1\right] \times\left[0, T_{\beta}^{k} 1\right]
$$

(see also Figure 3 for $m=4$ ), and define $\mathcal{T}_{\beta}: X \rightarrow X$ by

$$
\mathcal{T}_{\beta}(x, y):=\left(T_{\beta} x, \frac{1}{\beta}(\lfloor\beta x\rfloor+y)\right) .
$$

Let $Y:=[0,1] \times[0,1 / \beta]$ and $\mathcal{W}: Y \rightarrow Y$ the corresponding induced transformation under $\mathcal{T}_{\beta}$, that is

$$
\mathcal{W}(x, y)=\mathcal{T}_{\beta}^{n(x, y)}(x, y)
$$

where $n(x, y)=\min \left\{s>0: \mathcal{T}_{\beta}^{s}(x, y) \in Y\right\}$. Clearly one has

$$
\mathcal{W}(x, y)=\mathcal{T}_{\beta}^{k+1}(x, y),
$$

where $k=k(x) \in\{0,1, \ldots, m-1\}$ is such that $x \in\left(T_{\beta}^{m-k} 1, T_{\beta}^{m-k-1} 1\right]$. Notice that $\mathcal{W}$ maps rectangles to rectangles; see also Figure 3.

Finally, let $T_{\varepsilon}$ be the $(I, \varepsilon)$-GLS operator with partition $I$ given by $\left(T_{\beta}^{m-i} 1, T_{\beta}^{m-i-1} 1\right], 0 \leq i \leq m-1$ (see also Figure 3 ), and $\varepsilon(n)=0$ for

$\left({ }^{5}\right)$ For $m=2$ one has $\beta=(\sqrt{5}+1) / 2$, which is the golden mean. For $m \geq 3$ we call these $\beta$ 's pseudo golden mean numbers. 


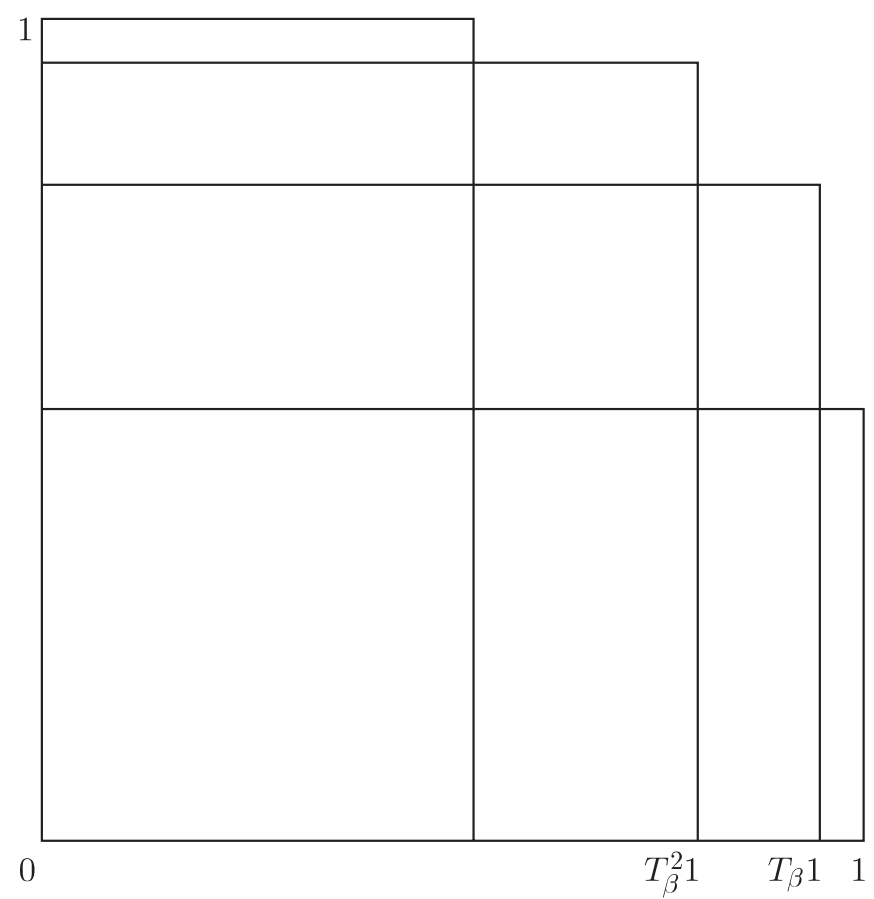

Fig. 3

each digit $n$. Notice that for $x \in\left(T_{\beta}^{m-i} 1, T_{\beta}^{m-(i+1)} 1\right], 0 \leq i \leq m-1$, one has

$$
T_{\varepsilon} x=T_{\beta}^{i+1} x .
$$

We have the following lemma.

Lemma 2. Let $\Psi:[0,1] \times[0,1] \rightarrow Y$ be defined by $\Psi(x, y):=(x, y / \beta)$. Then $\Psi$ is a measurable bijection which satisfies $\Psi \circ \mathcal{T}_{\varepsilon}=\mathcal{W} \circ \Psi$.

Proof. For $(x, y) \in[0,1] \times[0,1]$ let $i=i(x) \in\{0,1, \ldots, m-1\}$ be such that $x \in\left(T_{\beta}^{m-i} 1, T_{\beta}^{m-(i+1)} 1\right]$. From (14) it then follows that

$$
\mathcal{T}_{\varepsilon}(x, y)=\left(T_{\varepsilon} x, T_{\beta}^{m-i} 1+\frac{y}{\beta^{i+1}}\right]
$$

and therefore

$$
\Psi\left(\mathcal{T}_{\varepsilon}(x, y)\right)=\left(T_{\varepsilon} x, \frac{1}{\beta} T_{\beta}^{m-i} 1+\frac{y}{\beta^{i+2}}\right] .
$$

From (19), (20) and the definitions of $\mathcal{W}$ and $\Psi$ it now follows that $\mathcal{W}(\Psi(x, y))=\Psi\left(\mathcal{T}_{\varepsilon}(x, y)\right)$ for $i=0$ and in case $i \neq 0$ one has 


$$
\begin{aligned}
& \mathcal{W}(\Psi(x, y))=\mathcal{W}\left(x, \frac{y}{\beta}\right)=\mathcal{T}_{\beta}^{i+1}\left(x, \frac{y}{\beta}\right) \\
& \left.=\left(T_{\beta}^{i+1} x, \frac{1}{\beta}\left(0+(\underbrace{\frac{1}{\beta}\left(1+\ldots \frac{1}{\beta}\left(1+\frac{1}{\beta}(1\right.\right.}_{i \text { times }}+\frac{y}{\beta})\right) \ldots\right)\right) \\
& =\left(T_{\varepsilon} x, \frac{1}{\beta^{2}}+\ldots+\frac{1}{\beta^{i+1}}+\frac{y}{\beta^{i+2}}\right)=\Psi\left(\mathcal{T}_{\varepsilon}(x, y)\right) .
\end{aligned}
$$

Let $\varrho$ be the measure on $Y$ defined by

$$
\varrho(A):=(\lambda \times \lambda)\left(\Psi^{-1}(A)\right) \quad \text { for each Borel set } A \subset Y .
$$

It follows from Lemma 2 and the fact that $\lambda \times \lambda$ is an invariant measure for $\mathcal{T}_{\varepsilon}$ that $\varrho$ is invariant with respect to $\mathcal{W}$, and $\varrho=\beta(\lambda \times \lambda)$. Lemma 2 now at once yields the following proposition.

Proposition. The dynamical systems $\left([0,1] \times[0,1], \lambda \times \lambda, \mathcal{T}_{\varepsilon}\right)$ and $(Y, \varrho, \mathcal{W})$ are isomorphic.

Using standard techniques (see [CFS], p. 21) one obtains the measure $\mu$ on $X$ which is invariant with respect to $\mathcal{T}_{\beta}$, viz.

$$
\mu(A)=\frac{\beta}{\frac{1}{\beta}+\frac{2}{\beta^{2}}+\ldots+\frac{m}{\beta^{m}}}(\lambda \times \lambda)(A)
$$

for each Borel set $A \subset X$. One also easily shows that $\left(X, \mu, \mathcal{T}_{\beta}\right)$ forms the natural extension of $\left([0,1], \nu, T_{\beta}\right)$, where $\nu$ is the invariant measure with respect to $T_{\beta}$ [So]. Projecting $\mu$ on the first coordinate of $X$ yields $\nu$; one finds that $\nu$ has density $h(x)$, where

$$
h(x)=\frac{1}{\frac{1}{\beta}+\frac{2}{\beta}+\ldots+\frac{m}{\beta^{m}}} \sum_{x<T_{\beta}^{i} 1} \frac{1}{\beta^{i}},
$$

as given by $\mathrm{W}$. Parry $[\mathrm{Pa}]$.

Acknowledgements. We want to thank the referee for several helpful suggestions concerning the presentation of this paper.

\section{References}

[B] J. R. Brown, Ergodic Theory and Topological Dynamics, Academic Press, New York, 1976.

[BJW] W. Bosma, H. Jager and F. Wiedijk, Some metrical observations on the approximation by continued fractions, Indag. Math. 45 (1983), 281-299.

[CFS] I. P. Cornfeld, S. V. Fomin and Ya. G. Sinai, Ergodic Theory, Grundlehren Math. Wiss. 245, Springer, New York, 1982. 
[FS] C. Frougny and B. Solomyak, Finite beta-expansions, Ergodic Theory Dynamical Systems 12 (1992), 713-723.

[G] J. Galambos, Representations of Real Numbers by Infinite Series, Lecture Notes in Math. 502, Springer, Berlin, 1982.

[J] H. Jager, On decimal expansions, Zahlentheorie, Berichte aus dem Mathematische Forschungsinstitut Oberwolfach 5 (1971), 67-75.

[JdV] H. Jager and C. de Vroedt, Lüroth series and their ergodic properties, Indag. Math. 31 (1968), 31-42.

$\left[\mathrm{K}^{3} 1\right]$ S. Kalpazidou, A. Knopfmacher and J. Knopfmacher, Lüroth-type alternating series representations for real numbers, Acta Arith. 55 (1990), 311-322.

$\left[\mathrm{K}^{3} 2\right]--,-,-$, Metric properties of alternating Lüroth series, Portugal. Math. 48 (1991), 319-325.

[K] C. Kraaikamp, A new class of continued fraction expansions, Acta Arith. 57 (1991), 1-39.

[Li] P. Liardet, MR: 93m:11077.

$[\mathrm{Lu}]$ J. Lüroth, Ueber eine eindeutige Entwickelung von Zahlen in eine unendliche Reihe, Math. Ann. 21 (1883), 411-423.

[Pa] W. Parry, On the $\beta$-expansions of real numbers, Acta Math. Acad. Sci. Hungar. 11 (1960), 401-416.

[Pe] O. Perron, Irrationalzahlen, de Gruyter, Berlin, 1960.

[R] V. A. Rohlin, Exact endomorphisms of a Lebesgue space, Izv. Akad. Nauk SSSR Ser. Mat. 24 (1960) (in Russian); English translation: Amer. Math. Soc. Transl. Ser. 2, 39 (1969), 1-36.

[Sa] T. Šalát, Zur metrischen Theorie der Lürothschen Entwicklungen der reellen Zahlen, Czech. Math. J. 18 (1968), 489-522.

[So] B. Solomyak, Personal communication with C. Kraaikamp, Seattle, July 9, 1991.

[V] W. Vervaat, Success Epochs in Bernoulli Trails with Applications in Number Theory, Math. Centre Tracts 42, Amsterdam, 1972.

[W] P. Walters, An Introduction to Ergodic Theory, Grad. Texts in Math. 79, Springer, New York, 1982.

Mathematics Department

University of South Alabama

Mobile, Alabama 36688

U.S.A.

E-mail: jose@mathstat.usouthal.edu

Universiteit Utrecht

Department of Mathematics

Budapestlaan 6

P.O. Box 80.000

3508TA Utrecht, the Netherlands

E-mail: Dajani@math.ruu.nl
Department of Mathematics

University of Oregon Corvallis, Oregon 97331

U.S.A.

E-mail: burton@math.orst.edu

Technische Universiteit Delft TWI (SSOR)

Mekelweg 4

2628 CD Delft, the Netherlands

E-mail: C.Kraaikamp@twi.tudelft.nl

Received on 29.12.1994

and in revised form on 1.8.1995 